\title{
Einstein metrics, four-manifolds, and conformally Kähler geometry
}

\author{
Claude LeBrun
}

The Ricci curvature of a smooth Riemannian $n$-manifold $(M, g)$ is the function on the unit tangent bundle $U T M=\{v \in T M \mid g(v, v)=1\}$ given by

$$
v \mapsto r(v, v)
$$

where $r$ is the Ricci tensor of $g$. This function gives a precise measure of the volume distortion of the exponential map, since in geodesic normal coordinates the metric volume element becomes

$$
d \mu_{g}=\left[1-\frac{1}{6} r_{j k} x^{j} x^{k}+O\left(|x|^{3}\right)\right] d \mu_{\text {Euclidean }} .
$$

If the metric $g$ has constant Ricci curvature, we call it an Einstein metric, and $(M, g)$ is then said [4] to be an Einstein manifold. This of course happens precisely when $g$ satisfies the so-called Einstein equation

$$
r=\lambda g
$$

for some real constant $\lambda$. The number $\lambda$, which then represents the constant value of the Ricci curvature, is often called the Einstein constant of $(M, g)$. It is related to the scalar curvature $s=r_{i}{ }^{i}=\mathcal{R}^{i j}{ }_{i j}$ by $s=n \lambda$, so the Einstein constant and the scalar curvature in particular have the same sign.

All of this terminology is now completely standard among mathematicians, but Einstein himself would probably have been deeply uncomfortable with it. After all, mathematicians are primarily interested in equation (1) as a plausible avenue for geometrizing smooth compact manifolds. The fact that we are interested in Riemannian rather than Lorentzian solutions of (1) is simply not an issue for us, but it most certainly would have puzzled Einstein - especially insofar as we have adopted his notation $g$ for the metric, while forgetting that he intended this as an abbreviation for gravitational field. In any case, the supreme historical irony is perhaps that Einstein later [14] called equation (1) the "greatest mistake of his life," since the introduction

Supported in part by NSF grant DMS-0604735.

(C)2009 International Press 
of the so-called cosmological constant $\lambda$ into the gravitational field equations prevented him [31] from predicting the observed Hubble expansion of the universe.

Of course, we mathematicians have a long history of latching onto good ideas, without worrying terribly much about where they came from. This is nothing new. Indeed, it has been nearly two centuries since Goethe [15] complained that, "Mathematicians are like Frenchmen; you tell them something, they translate it into their own language, and, before you know, it's something entirely different."

In any case, one of the central problems of modern differential geometry is to determine precisely which smooth compact $n$-manifolds admit Einstein metrics. When $n=2$ or 3 , the Einstein metrics are just the metrics of constant sectional curvature, so when such a metric exists, it geometrizes the manifold in the extremely strong sense of displaying it as a quotient of a standard, homogeneous model by a discrete group of isometries. In fact, the existence of such metrics on any 2-manifold is guaranteed by the classical uniformization theorem. By contrast, not every compact 3-manifold admits an Einstein metric; but Perelman's successful attack $[\mathbf{2 0 , 3 4 , 3 5 , 3 6 ]}$ on the Thurston geometrization program via the Hamilton Ricci flow [18] has still taught us that every 3-manifold can at least be broken up into Einstein and collapsed pieces. Dimension four represents an important transition for equation (1); when $n=4$, Einstein metrics are usually no longer locally homogeneous, but special low-dimensional phenomena, discussed below, nonetheless provide powerful links between their geometry and the differential topology of the underlying manifold. On the other hand, when $n \geq 5$, Einstein metrics do not seem to offer a plausible geometrization of manifolds, because $[\mathbf{5}, \mathbf{6}]$ even familiar manifolds like high-dimensional spheres typically admit unitvolume Einstein metrics for many different values of the Einstein constant $\lambda$.

The case of $n=4$ thus seems particularly interesting and important. But unfortunately, we are still far from being able to determine precisely which smooth compact 4-manifolds $M^{4}$ admit Einstein metrics. Nevertheless, Kähler geometry provides a rich source of examples of Einstein metrics on compact 4-manifolds, and Seiberg-Witten theory allows one to mimic Kähler geometry when treating even non-Kähler metrics on compact complex surfaces. This article will therefore focus on the following restricted version of the problem:

QUESTION 1. If $M^{4}$ is the underlying smooth manifold of a compact complex surface $(M, J)$, when does $M$ carry an Einstein metric?

It turns out that there is a powerful analogy between complex surfaces and 4-manifolds that carry symplectic forms (closed, non-degenerate 2 -forms). It is therefore natural to also ask

QUESTION 2. If $M^{4}$ is a smooth compact 4-manifold that admits a symplectic form $\omega$, when does $M$ carry an Einstein metric? 
Without some restriction on the Einstein metric, a full answer to even these restricted questions remains tantalizingly out of reach. However, if we are willing to also make an assumption about the sign of the Einstein constant $\lambda$, definitive answers like the following [9] can now be given.

THEOREM 1. Suppose that $M$ is a smooth compact oriented 4-manifold which admits a complex structure $J$. Then $M$ also admits a (possibly unrelated) Einstein metric $g$ with $\lambda>0$ if and only if $M$ appears on the following list of diffeotypes:

$$
M \approx\left\{\begin{array}{l}
\mathbb{C P}_{2} \# k \overline{\mathbb{C P}}_{2}, \quad 0 \leq k \leq 8, \\
\text { or } \\
S^{2} \times S^{2}
\end{array}\right.
$$

Here $\overline{\mathbb{C P}}_{2}$ denotes the smooth oriented manifold obtained by giving $\mathbb{C P}_{2}$ the non-standard orientation, and

$$
\mathbb{C P}_{2} \# k \overline{\mathbb{C P}}_{2}=\mathbb{C P}_{2} \# \underbrace{\overline{\mathbb{P P}}_{2} \# \cdots \# \overline{\mathbb{C P}}_{2}}_{k},
$$

where \# indicates the connected sum operation, which glues two oriented 4-manifolds together by first removing a standard ball from each, and then identifying the resulting boundary spheres via a reflection. The relevance of this operation to complex geometry arises from the fact that if $N$ is a complex surface, we may may replace any point $p \in N$ with a $\mathbb{C P}_{1}$ of selfintersection -1 to obtain a new complex surface $\hat{N}$, called the blow-up of $N$ at $p$, which is diffeomorphic to $N \# \overline{\mathbb{C P}}_{2}$. Thus the diffeotypes listed above can be realized by $\mathbb{C P}_{1} \times \mathbb{C P}_{1}$ and of $\mathbb{C P}_{2}$ blown up at $k$ points in general position, $0 \leq k \leq 8$. In other words $[\mathbf{1 1}, \mathbf{2 7}]$, this list describes the diffeotypes of the Del Pezzo surfaces, which are by definition the compact complex surfaces which are Fano, in the sense that $c_{1}^{\mathbb{R}} \in H^{2}(M, \mathbb{R})$ is a Kähler class.

If we broaden the question by merely requiring that the Einstein constant be non-negative, more diffeotypes are allowed, but a complete classification [24] can still be given.

TheOREM 2. Suppose that $M$ is a smooth compact oriented 4-manifold which admits an integrable complex structure $J$. Then $M$ also admits an Einstein metric $g$ with $\lambda \geq 0$ if and only if $M$ appears on the following list of diffeotypes:

$$
M \approx\left\{\begin{array}{l}
\mathbb{C P}_{2} \# k \overline{\mathbb{C P}}_{2}, 0 \leq k \leq 8 \\
S^{2} \times S^{2} \\
K 3 \\
K 3 / \mathbb{Z}_{2} \\
T^{4} \\
T^{4} / \mathbb{Z}_{2}, T^{4} / \mathbb{Z}_{3}, T^{4} / \mathbb{Z}_{4}, T^{4} / \mathbb{Z}_{6}, \\
T^{4} /\left(\mathbb{Z}_{2} \oplus \mathbb{Z}_{2}\right), T^{4} /\left(\mathbb{Z}_{3} \oplus \mathbb{Z}_{3}\right), \text { or } T^{4} /\left(\mathbb{Z}_{2} \oplus \mathbb{Z}_{4}\right)
\end{array}\right.
$$


The diffeotypes we have added in passing from Theorem 1 to Theorem 2 are exactly those represented by the compact complex surfaces of Kähler type for which $c_{1} \in H^{2}(M, \mathbb{Z})$ is a torsion class. These are traditionally sorted into four baskets $[\mathbf{3}, \mathbf{1 6}]$. The first basket holds the $K 3$ surfaces, named in honor of Kummer, Kähler, and Kodaira, and defined to be the simply connected compact complex surfaces with $c_{1}=0$; they are all deformation equivalent [21], and so, for example, are all diffeomorphic to the smooth quartic $t^{4}+u^{4}+v^{4}+w^{4}=0$ in $\mathbb{C P}_{3}$. Next, there are the Enriques surfaces, which are $\mathbb{Z}_{2}$-quotients of $K 3$ surfaces; again, there is only one diffeotype. Then there are the Abelian surfaces, which are diffeomorphic to the 4-torus $T^{4}$. And finally, there are the hyper-elliptic surfaces, which are quotients of $T^{4}$ by one of seven finite groups of affine motions, each of which acts in a uniquely specified way.

Symplectic analogs of these results are also true:

THEOREM 3. Suppose that $M$ is a smooth compact oriented 4-manifold which admits a symplectic structure $\omega$. Then $M$ also admits an Einstein metric $g$ with $\lambda>0$ iff it is diffeomorphic to one of the manifolds listed in Theorem 1. Similarly, it admits an Einstein metric $g$ with $\lambda \geq 0$ iff it is diffeomorphic to one of the manifolds listed in Theorem 2.

The proofs of these theorems proceed on two distinct fronts: existence results for Einstein metrics; and obstructions to the existence of Einstein metrics. We will first discuss the relevant existence results. The main ideas needed for these arise from Kähler geometry and conformal geometry.

Recall that a Riemannian metric on a connected $2 m$-manifold $M$ is Kähler iff its holonomy group is (conjugate to) a subgroup of $U(m) \subset O(2 m)$. This is equivalent to saying there exists an almost complex structure $J \in \Gamma($ End $(T M)), J^{2}=-1$, with $\nabla J=0$ and $g(J \cdot, J \cdot)=g$. When this happens, $J$ is integrable, and $(M, J)$ thus becomes a complex manifold. Moreover, the $J$-invariant 2 -form $\omega$ defined by $\omega=g(J \cdot, \cdot)$, called the Kähler form of $(M, g, J)$, satisfies $d \omega=0$. In particular, $\omega$ is a a symplectic form on $M$, meaning that it is a closed 2 -form of maximal rank. One of the magical features of Kähler geometry is that the 2-form defined by $\operatorname{ir}(J \cdot, \cdot)$ is exactly the curvature of the canonical line bundle $K=\Lambda^{m, 0}$, where $m$ is the complex dimension. Note that $m=2$ in the $n=4$ case that will concern us here.

We will also need some rudiments of conformal geometry. Recall that two Riemannian metrics $g$ and $h$ are said to be conformally related if $g=f h$ for some smooth function $f: M \rightarrow \mathbb{R}^{+}$. If $h$ is also a Kähler metric, we will then say that the metric $g$ is conformally Kähler. When the complex dimension $m$ is at least two, and if $f$ is non-constant, then $g$ and $h$ can then never be Kähler metrics adapted to the same complex structure $J$. However, it is worth pointing out that there are some rare but interesting examples with $m=2$ where $g$ and $h$ are both Kähler metrics, but are adapted to different complex structures $J$ and $\tilde{J}$. 
Many of the existence results needed here are supplied by the theory of Kähler-Einstein metrics (that is, of Einstein metrics that happen to be Kähler). The foundations of this theory were laid by Calabi [7], who translated the problem into a non-linear scalar PDE, called the complex Monge-Ampère equation, and conjectured that a compact complex manifold of Kähler type with $c_{1}{ }^{\mathbb{R}}=0$ would admit a unique Ricci-flat Kähler metric in each Kähler class. Yau's proof $[\mathbf{4 2}, \mathbf{4 3}]$ of this conjecture remains a major landmark of modern differential geometry. It predicts, in particular, that both $K 3$ and the Enriques surface $K 3 / \mathbb{Z}_{2}$ admit KählerEinstein metrics with $\lambda=0$. Of course, $T^{4}$ and its relevant quotients also admit Ricci-flat metrics, but in these cases the metrics are actually flat, and so can be constructed directly, without the use of any sophisticated machinery.

The theory of Kähler-Einstein metrics is considerably more subtle when $\lambda>0$, but case-by-case investigations by Siu [37] and Tian-Yau [40] did reveal that there exist $\lambda>0$ Kähler-Einstein metrics on $\mathbb{C P}_{2} \# k \overline{\mathbb{C P}}_{2}$ for each $k \in\{3, \ldots, 8\}$. Of course, $\mathbb{C P}_{2}$ and $S^{2} \times S^{2}$ also admit such metrics, but in these cases the relevant metrics are just the obvious homogeneous ones.

By contrast, however, $\mathbb{C P}_{2} \# \overline{\mathbb{C P}}_{2}$ and $\mathbb{C P}_{2} \# 2 \overline{\mathbb{C P}}_{2}$ cannot admit KählerEinstein metrics. This reflects an important observation due to Matsushima [29]. Namely, if a compact complex manifold $(M, J)$ admits a KählerEinstein metric $g$ with $\lambda>0$, then its biholomorphism group $\operatorname{Aut}(M, J)$ must be a reductive Lie group, since the identity component $\operatorname{Isom}_{0}(M, g)$ of the isometry group is then a compact real form for $\operatorname{Aut}_{0}(M, J)$. Since $\mathbb{C P}_{2} \# \overline{\mathbb{C P}}_{2}$ and $\mathbb{C P}_{2} \# 2 \overline{\mathbb{C P}}_{2}$ have non-reductive automorphism groups, this therefore implies that they cannot admit Kähler-Einstein metrics.

Nonetheless, in what was long thought to be an entirely unrelated development, Page [33] had succeeded in constructing an explicit $\lambda>0$ Einstein metric on $\mathbb{C P}_{2} \# \overline{\mathbb{C P}}_{2}$ by a very different method. The Page metric is of cohomogeneity one, meaning that its isometry group has a family of hypersurfaces as orbits. This feature allowed Page to construct his metric by solving an appropriate ODE. While none of this seemed to have anything to do with Kähler geometry, Derdziński [12] later discovered that the Page metric is actually conformally Kähler, and, in the same paper, then went on to prove a number of fundamental results concerning conformally Kähler, Einstein metrics on 4-manifolds.

Recently, in joint work [9] with Xiuxiong Chen and Brian Weber, the present author managed to prove the existence of a companion of the Page metric. Namely, there is a conformally Kähler, $\lambda>0$ Einstein metric $g$ on $\mathbb{C P}_{2} \# 2 \overline{\mathbb{C P}}_{2}$. This metric is toric, and so of cohomogeneity two, but it is not constructed explicitly. Roughly speaking, the metric is found by first minimizing the functional

$$
\mathcal{A}(h)=\int_{M} s^{2} d \mu_{h}
$$


on the space of all Kähler metrics $h$ compatible with the fixed complex structure $J$, where $s$ denotes the scalar curvature of $h$. Here it is crucial that the Kähler class $[\omega]$ of $h$ is allowed to vary in this problem. If, by contrast, we fixed $[\omega]$, and only considered Kähler metrics with Kähler form in this fixed de Rham class, we would instead be talking about Calabi's problem for extremal Kähler metrics [8]. Thus, the problem under discussion here really amounts to minimizing $\mathcal{A}(h)$ among extremal Kähler metrics $h$. One thus proceeds by restricting $\mathcal{A}$ to the set of extremal Kähler metrics, and showing that a critical point $h$ exists for this problem. This preferred extremal Kähler metric turns out to have scalar curvature $s>0$, and one is therefore able to define a new Riemannian metric by setting $g=s^{-2} h$. The punch line is that this conformally Kähler metric $g$ then actually turns out to be Einstein, with $\lambda>0$.

To explain this seeming miracle, we will need a bit more background regarding 4-dimensional Riemannian geometry. The special nature of dimension four basically stems from the fact that the bundle $\Lambda^{2}$ of 2 -forms over an oriented Riemannian 4-manifold $(M, g)$ decomposes, in a conformally invariant manner, into a direct sum

$$
\Lambda^{2}=\Lambda^{+} \oplus \Lambda^{-}
$$

of the self-dual and anti-self-dual 2-forms; here $\Lambda^{ \pm}$are by definition the $( \pm 1)$-eigenspaces of the Hodge star operator. Since the Riemann curvature tensor may be thought of as a self-adjoint linear map

$$
\mathcal{R}: \Lambda^{2} \rightarrow \Lambda^{2}
$$

it can therefore be decomposed into irreducible pieces

$$
\mathcal{R}=\left(\begin{array}{c|c}
W_{+}+\frac{s}{12} & \stackrel{\circ}{r} \\
\hline \stackrel{\circ}{r} & W_{-}+\frac{s}{12}
\end{array}\right)
$$

where $s$ is the scalar curvature, $\stackrel{\circ}{r}=r-\frac{s}{4} g$ is the trace-free Ricci curvature, and where $W_{ \pm}$are the trace-free pieces of the appropriate blocks. The tensors $W_{ \pm}$are both conformally invariant, and are respectively called the selfdual and anti-self-dual Weyl curvature tensors. Their sum $W=W_{+}+W_{-}$is called the Weyl tensor, and is exactly the conformally invariant part of the curvature tensor $\mathcal{R}$.

We can now consider the conformally invariant functional

$$
\mathcal{W}(g)=\int_{M}|W|^{2} d \mu_{g}=\int_{M}\left(\left|W_{+}\right|^{2}+\left|W_{-}\right|^{2}\right) d \mu_{g}
$$


whose gradient on the space of metrics is represented [4] by the Bach tensor $B$, which is the traceless divergence-free tensor field given by

$$
B_{a b}:=\left(\nabla^{c} \nabla^{d}+\frac{1}{2} r^{c d}\right) W_{a c b d} .
$$

This tensor automatically vanishes for any conformally Einstein metric, since an Einstein metric is certainly a critical point of both non-Weyl contributions to the 4-dimensional Gauss-Bonnet formula

$$
\chi(M)=\frac{1}{8 \pi^{2}} \int_{M}\left(|W|^{2}+\frac{s^{2}}{24}-\frac{|\grave{r}|^{2}}{2}\right) d \mu .
$$

But since the signature

$$
\tau(M)=\frac{1}{12 \pi^{2}} \int_{M}\left(\left|W_{+}\right|^{2}-\left|W_{-}\right|^{2}\right) d \mu
$$

is also a topological invariant, $\mathcal{W}$ differs from twice the functional

$$
\mathcal{W}_{+}(g)=\int_{M}\left|W_{+}\right|^{2} d \mu_{g}
$$

by only a constant, and the Bach tensor can correspondingly also be expressed as

$$
B_{a b}:=2\left(\nabla^{c} \nabla^{d}+\frac{1}{2} \stackrel{r}{r}^{c d}\right)\left(W_{+}\right)_{a c b d}
$$

Now, both of these last observations have rather dramatic consequences in the Kähler context. First, since

$$
\left|W_{+}\right|^{2}=\frac{s^{2}}{24}
$$

for any Kähler metric on a 4-manifold, the critical points of the functional $\mathcal{A}$ coincide with the critical points of the restriction of $\mathcal{W}$ to the space of Kähler metrics, and are therefore precisely those extremal Kähler metrics $h$ for which the Bach tensor $B$ is $L^{2}$-orthogonal to all infinitesimal variations through Kähler metrics. Second, because $W_{+}$of a Kähler metric can be written in terms of the scalar curvature and Kähler form, the Bach tensor of an extremal Kähler metric $h$ can explicitly be expressed $[\mathbf{9}, \mathbf{1 2}]$ as

$$
B=\frac{1}{12}\left[s \stackrel{\circ}{r}+2 \operatorname{Hess}_{0}(s)\right]
$$

and therefore corresponds to a primitive harmonic $(1,1)$-form

$$
\psi=B(J \cdot, \cdot)=\frac{1}{12}[s \rho+2 i \partial \bar{\partial} s]_{0} .
$$

This implies that $B$ is actually tangent to a curve of Kähler metrics $h+t B$. Hence the critical points of the functional $\mathcal{A}$ are exactly the Bach-flat Kähler metrics, meaning those Kähler metrics for which $B=0$. Since multiplying a 4-dimensional metric by $u^{2}$ alters its traceless Ricci tensor by

$$
\stackrel{\circ}{r} \rightsquigarrow \hat{r}=\stackrel{r}{r}-2 u \operatorname{Hess}_{0}\left(u^{-1}\right)
$$


we also see that, for any extremal Kähler metric $h$ on a complex surface, the conformally related metric $g=s^{-2} h$ will have traceless Ricci curvature

$$
\hat{r}=12 s^{-1} B
$$

where $B$ is the Bach tensor of $h$. Thus, any Bach-flat Kähler metric will be conformal to an Einstein metric, at least on the open set where $s \neq 0$.

Fortunately, the $\mathcal{A}$-energy of an extremal Kähler metric is a function of the Kähler class $[\omega]$ which can be calculated a priori, without even knowing whether or not the extremal metric actually exists; namely it is given by

$$
\mathcal{A}_{\text {virtual }}=32 \pi^{2} \frac{\left(c_{1} \cdot[\omega]\right)^{2}}{[\omega]^{2}}+\left\|\mathcal{F}_{[\omega]}\right\|^{2}
$$

where $\mathcal{F}$ is Futaki invariant [13]. This allows one, at the very outset, to locate the target Kähler class [ $\omega]$ where the minimizer $h$ ought to live. The intimate relationship between the Futaki invariant and the scalar curvature $s$ also allows one to show that, if the target extremal Kähler metric $h$ exists, then it has $s>0$, so our Einstein metric $g=s^{-2} h$ really will then be defined on all of $M=\mathbb{C P}_{2} \# 2 \overline{\mathbb{C P}}_{2}$.

Now a gluing argument of Arezzo, Pacard, and Singer [1] implies that $\mathbb{C P}_{2} \# 2 \overline{\mathbb{C P}}_{2}$ does admit some extremal Kähler metrics, albeit near the edge of the Kähler cone and far from the target class. On the other hand, a quite general implicit-function-theorem argument [25] shows that the Kähler classes of extremal Kähler metrics form an open subset of the Kähler cone. To prove the existence of the preferred extremal metric $h$, it therefore suffices to choose a nice path in the Kähler cone from a class where one has existence to the target class $[\omega]$, and show that the the set of classes along this path with extremal representatives is closed as well as open. To do this, one appeals to a weak compactness result for extremal Kähler metrics [10], which allows one to conclude that sequences of such metrics have subsequences which Gromov-Hausdorff converge to orbifolds, once uniform Sobolev and energy bounds have been established. Smooth convergence is then established by ruling out all possible bubbling modes, using energy bounds and topological arguments. Finally, toric geometry is used to show that the limit Kähler metric is compatible with the original complex structure, and belongs to the expected Kähler class.

These existence results suffice to prove one direction of implication in Theorems 1, 2, and 3. To prove the converse statements, one instead needs to consider obstructions to the existence of Einstein metrics.

The first such result that we will need is the Hitchin-Thorpe inequality [19]. This is obtained by observing that the Gauss-Bonnet and signature formulas together imply that

$$
(2 \chi+3 \tau)(M)=\frac{1}{4 \pi^{2}} \int_{M}\left(\frac{s^{2}}{24}+2\left|W_{+}\right|^{2}-\frac{|\stackrel{\circ}{r}|^{2}}{2}\right) d \mu_{g} .
$$


Since Einstein metrics are characterized by $\stackrel{\circ}{r}=0$, the existence of such a metric would make the integrand in the above expression non-negative, so a smooth compact oriented 4-manifold can only admit an Einstein metric $g$ if $(2 \chi+3 \tau)(M) \geq 0$, with equality iff $g$ is Ricci-flat and anti-self-dual $\left(W_{+} \equiv 0\right)$. The latter happens, however, iff $(M, g)$ has reduced holonomy $\subset S U(2)$. If $M$ admits a complex or symplectic structure, this then implies [24] that the relevant structure has $c_{1}^{2} \geq 0$, with equality iff $M$ is diffeomorphic to a complex surface with $c_{1}$ torsion and $b_{1}$ even. For the purpose of proving Theorems 1, 2, and 3, one may thus assume henceforth that $c_{1}^{2}(M)>0$.

The rest of the proof depends on Seiberg-Witten theory, which allows one to imitate certain aspects of Kähler geometry when discussing nonKähler metrics on appropriate 4-manifolds. One can't hope to generalize the $\bar{\partial}$ operator in this setting, but $\bar{\partial}+\bar{\partial}^{*}$ does have a natural generalization, namely as a $\operatorname{spin}^{c}$ Dirac operator.

Thus, suppose that $M$ is a smooth compact 4-manifold which admits an almost-complex structure $J$, which we then use to orient $M$. Let $L=\Lambda^{0,2}$ be the anti-canonical line bundle of $J$. For any metric $g$ on $M$, the bundles

$$
\begin{aligned}
& \mathbb{V}_{+}=\Lambda^{0,0} \oplus \Lambda^{0,2} \\
& \mathbb{V}_{-}=\Lambda^{0,1}
\end{aligned}
$$

can then formally be written as

$$
\mathbb{V}_{ \pm}=\mathbb{S}_{ \pm} \otimes L^{1 / 2}
$$

where $\mathbb{S}_{ \pm}$are the left- and right-handed spinor bundles of $g$. Each unitary connection $A$ on $L$ then induces a $\operatorname{spin}^{c}$ Dirac operator

$$
D_{A}: \Gamma\left(\mathbb{V}_{+}\right) \rightarrow \Gamma\left(\mathbb{V}_{-}\right)
$$

generalizing $\bar{\partial}+\bar{\partial}^{*}$. The Seiberg-Witten equations [41] are the coupled system

$$
\begin{aligned}
D_{A} \Phi & =0 \\
F_{A}^{+} & =-\frac{1}{2} \Phi \odot \bar{\Phi}
\end{aligned}
$$

for the unknowns $A$ and $\Phi \in \Gamma\left(\mathbb{V}_{+}\right)$, where $F_{A}^{+}$denotes the self-dual part of the curvature of $A$. These equations are non-linear, but become elliptic once one imposes the 'gauge-fixing' condition

$$
d^{*}\left(A-A_{0}\right)=0
$$

to eliminate automorphisms of $L \rightarrow M$. Because the Seiberg-Witten equations imply the Weitzenböck formula

$$
0=2 \Delta|\Phi|^{2}+4\left|\nabla_{A} \Phi\right|^{2}+s|\Phi|^{2}+|\Phi|^{4}
$$


one can show that the moduli space of solutions is compact. In the presence of the assumption that $c_{1}^{2}(M, J)>0$, one can define the Seiberg-Witten invariant by counting solutions of the Seiberg-Witten equations, modulo gauge equivalence and with appropriate multiplicities. This count is then independent of the metric. However, if there exists a metric $g$ of scalar curvature $s \geq 0$, and if $c_{1}^{2}(M, J)>0$, the above Weitzenböck formula forces the non-existence of solutions for the given metric, so the Seiberg-Witten invariant must then vanish.

By contrast, the Seiberg-Witten invariant would be non-zero for a complex surface of general type $[\mathbf{2 2}, \mathbf{3 2}, \mathbf{4 1}]$, so the Kodaira classification $[\mathbf{3}]$ allows us to conclude that a complex surface with $c_{1}^{2}>0$ can therefore only admit a Riemannian metric of non-negative scalar curvature if it is deformation equivalent to a Del Pezzo surface. The converse directions in Theorems 1 and 2 now follow. In the symplectic case, one may reach the analogous conclusion by appealing to a result of Liu [26]. Liu's argument rests in part on a result of McDuff [30], which characterizes rational symplectic manifolds by the presence of a pseudo-holomorphic 2-sphere of positive selfintersection. The other crucial ingredient is a theorem of Taubes [38], which produces pseudo-holomorphic curves from solutions of perturbed versions of the Seiberg-Witten equations for appropriate $\operatorname{spin}^{c}$ structures. The converse direction in Theorem 3 thus also follows, as advertised.

While we now know that all the manifolds listed in Theorem 2 actually admit Einstein metrics, there are still open questions regarding the moduli of such metrics. Our understanding is quite complete in the cases of $K 3, T^{4}$, and their quotients, as these spaces saturate the Hitchin-Thorpe inequality; every Einstein metric on any such manifold is therefore locally hyper-Kähler, and one can therefore $[\mathbf{3}]$ in particular show that the moduli space of Einstein metrics on any of these manifolds is connected. But the Del Pezzo cases are quite a different story. For example, while we do have a reasonable understanding of the moduli of Kähler-Einstein metrics on Del Pezzo surfaces [39], nothing we know precludes the existence of other components of the moduli space; however, when a Kähler-Einstein metric exists, it is at least known [17] that any non-Kähler Einstein metric would necessarily have strictly smaller Einstein-Hilbert action. By contrast, the Page and Chen-LeBrun-Weber metrics are not even currently known to have such a maximizing property. Indeed, the uniqueness of the latter metric has not really been conclusively demonstrated even among conformally Kähler metrics, although computer-based calculations [28] lend enormous credibility to such an assertion.

What about the $\lambda<0$ case? The Aubin/Yau existence theorem $[\mathbf{2 , 4 2}]$ constructs Kähler-Einstein metrics with $\lambda<0$ on a profusion of minimal complex surfaces of general type. But in the converse direction, we only have some partial results. If $(M, J)$ is a compact complex surface, and if the underlying smooth 4-manifold $M$ admits an Einstein metric $g$, then it is easy to show, using the Hitchin-Thorpe inequality and the Kodaira classification, 
that either $M$ appears on the list in Theorem 2, or else that $(M, J)$ is of general type. What remains unknown is whether the underlying 4-manifold of a non-minimal complex surface of general type can ever admit an Einstein metric. The best we can currently say is that a surface of general type which admits an Einstein metric cannot be 'too' non-minimal, in the following numerical sense [23]: if $X$ is a minimal complex surface of general type, then its $k$-point blow-up $X \# k \overline{\mathbb{C P}}_{2}$ cannot admit Riemannian Einstein metrics if $k \geq c_{1}^{2}(X) / 3$. Analogous results can also be proved in the symplectic setting. But, basically, our knowledge of the $\lambda<0$ realm remains frustratingly incomplete, even though it is precisely here that most of the known examples reside. Perhaps what we really need now is some major progress in constructing Einstein metrics that have nothing to do with Kähler geometry!

\section{References}

[1] C. Arezzo, F. Pacard, And M. Singer, Extremal metrics on blow ups. e-print math.DG/0701028, 2007.

[2] T. Aubin, Equations du type Monge-Ampère sur les variétés kählériennes compactes, C. R. Acad. Sci. Paris, 283A (1976), pp. 119-121.

[3] W. Barth, C. Peters, and A. Van de Ven, Compact complex surfaces, vol. 4 of Ergebnisse der Mathematik und ihrer Grenzgebiete (3) [Results in Mathematics and Related Areas (3)], Springer-Verlag, Berlin, 1984.

[4] A. L. Besse, Einstein manifolds, vol. 10 of Ergebnisse der Mathematik und ihrer Grenzgebiete (3) [Results in Mathematics and Related Areas (3)], Springer-Verlag, Berlin, 1987.

[5] C. Bӧнм, Inhomogeneous Einstein metrics on low-dimensional spheres and other low-dimensional spaces, Invent. Math., 134 (1998), pp. 145-176.

[6] C. P. Boyer and K. Galicki, Sasakian geometry, Oxford Mathematical Monographs, Oxford University Press, Oxford, 2008.

[7] E. CALABI, On Kähler manifolds with vanishing canonical class, in Algebraic geometry and topology. A symposium in honor of S. Lefschetz, Princeton University Press, Princeton, N.J., 1957, pp. 78-89.

[8] — Extremal Kähler metrics, in Seminar on Differential Geometry, vol. 102 of Ann. of Math. Stud., Princeton Univ. Press, Princeton, N.J., 1982, pp. 259-290.

[9] X. X. Chen, C. LeBrun, and B. Weber, On conformally Kähler, Einstein manifolds, J. Amer. Math. Soc., 21 (2008), pp. 1137-1168.

[10] X. X. Chen And B. Weber, Moduli spaces of critical Riemannian metrics with $L^{n / 2}$ norm curvature bounds. e-print arXiv:0705.4440, 2007.

[11] M. Demazure, Surfaces de del Pezzo, II, III, IV, V, in Séminaire sur les Singularités des Surfaces, vol. 777 of Lecture Notes in Mathematics, Berlin, 1980, Springer, pp. 21-69.

[12] A. Derdziński, Self-dual Kähler manifolds and Einstein manifolds of dimension four, Compositio Math., 49 (1983), pp. 405-433.

[13] A. Futaki And T. Mabuchi, Bilinear forms and extremal Kähler vector fields associated with Kähler classes, Math. Ann., 301 (1995), pp. 199-210.

[14] G. Gamow, My World Line; an Informal Autobiography, Viking Press, New York, NY, 1970.

[15] J. W. V. Goethe, Maximen und Reflektionen, 1833/1840. Republished on-line at http://www.wissen-im-netz.info/literatur/goethe/maximen. 
[16] P. Griffiths And J. Harris, Principles of Algebraic Geometry, Wiley-Interscience, New York, 1978.

[17] M. J. GuRsky, The Weyl functional, de Rham cohomology, and Kähler-Einstein metrics, Ann. of Math. (2), 148 (1998), pp. 315-337.

[18] R. Hamilton, Three-manifolds with positive Ricci curvature, J. Differential Geom., 17 (1982), pp. 255-306.

[19] N. J. Hitchin, On compact four-dimensional Einstein manifolds, J. Differential Geom., 9 (1974), pp. 435-442.

[20] B. Kleiner AND J. LotT, Notes on Perelman's papers. e-print math.DG/0605667.

[21] K. Kodaira, On the structure of compact complex analytic surfaces. I, Amer. J. Math., 86 (1964), pp. 751-798.

[22] C. LeBrun, Four-manifolds without Einstein metrics, Math. Res. Lett., 3 (1996), pp. $133-147$.

[23] - Ricci curvature, minimal volumes, and Seiberg-Witten theory, Inv. Math., 145 (2001), pp. 279-316.

[24] C. LEBrun, Einstein metrics, complex surfaces, and symplectic 4-manifolds, Math. Proc. Cambr. Phil. Soc., 147 (2009), pp. 1-8. e-print arXiv:0803.3743[math.DG].

[25] C. LeBrun and S. R. Simanca, On the Kähler classes of extremal metrics, in Geometry and Global Analysis (Sendai, 1993), Tohoku Univ., Sendai, 1993, pp. 255-271.

[26] A.-K. LiU, Some new applications of general wall crossing formula, Gompf's conjecture and its applications, Math. Res. Lett., 3 (1996), pp. 569-585.

[27] Y. I. Manin, Cubic Forms: Algebra, Geometry, Arithmetic, North-Holland Publishing Co., Amsterdam, 1974. Translated from the Russian by M. Hazewinkel.

[28] G. Maschler, Uniqueness of Einstein metrics conformal to extremal Kähler metricsa computer assisted approach, AIP Conf. Proc., 1093 (2009), pp. 132-143. On-line at http://link.aip.org/link/?APCPCS/1093/132/1.

[29] Y. Matsushima, Sur la structure du groupe d'homéomorphismes d'une certaine varieté Kählérienne, Nagoya Math. J., 11 (1957), pp. 145-150.

[30] D. McDuff, The structure of rational and ruled symplectic 4-manifolds, J. Amer. Math. Soc., 3 (1990), pp. 679-712.

[31] C. W. Misner, K. S. Thorne, and J. A. Wheeler, Gravitation, W. H. Freeman and Co., San Francisco, Calif., 1973.

[32] J. Morgan, The Seiberg-Witten Equations and Applications to the Topology of Smooth Four-Manifolds, vol. 44 of Mathematical Notes, Princeton University Press, 1996.

[33] D. PAGE, A compact rotating gravitational instanton, Phys. Lett., 79B (1979), pp. 235-238.

[34] G. Perelman, The entropy formula for the Ricci flow and its geometric applications. e-print math.DG/0211159.

[35] - Finite extinction time for the solutions to the Ricci flow on certain threemanifolds. e-print math.DG/0307245.

[36] — Ricci flow with surgery on three-manifolds. e-print math.DG/0303109.

[37] Y. Siu, The existence of Kähler-Einstein metrics on manifolds with positive anticanonical line bundle and suitable finite symmetry group, Ann. Math., 127 (1988), pp. 585-627.

[38] C. H. Taubes, The Seiberg-Witten and Gromov invariants, Math. Res. Lett., 2 (1995), pp. 221-238.

[39] G. Tian, On Calabi's conjecture for complex surfaces with positive first Chern class, Inv. Math., 101 (1990), pp. 101-172.

[40] G. Tian and S. T. YAU, Kähler-Einstein metrics on complex surfaces with $\mathbf{c}_{1}>0$, Comm. Math. Phys., 112 (1987), pp. 175-203.

[41] E. Witten, Monopoles and four-manifolds, Math. Res. Lett., 1 (1994), pp. 809-822. 
[42] S. T. YAU, Calabi's conjecture and some new results in algebraic geometry, Proc. Nat. Acad. USA, 74 (1977), pp. 1789-1799.

[43] - On the Ricci curvature of a compact Kähler manifold and the complex MongeAmpère equation. I, Comm. Pure Appl. Math., 31 (1978), pp. 339-411.

Department of Mathematics, SUny at Stony Brook, Stony Brook, Ny 11794-3651

E-mail address: claude@math.sunysb.edu 
